\title{
BMJ Open Well-being among survivors of out-of- hospital cardiac arrest: a cross-sectional retrospective study in Sweden
}

\author{
Adam Viktorisson, ${ }^{1}$ Katharina S Sunnerhagen, ${ }^{1}$ Ulrika Pöder, ${ }^{2}$ Johan Herlitz, ${ }^{3,4}$ \\ Åsa B Axelsson ${ }^{5}$
}

To cite: Viktorisson $A$,

Sunnerhagen KS, Pöder U, et al. Well-being among survivors of out-of-hospital cardiac arrest: a cross-sectional retrospective study in Sweden. BMJ Open 2018;8:e021729. doi:10.1136/ bmjopen-2018-021729

- Prepublication history for this paper is available online. To view these files, please visit the journal online (http://dx.doi. org/10.1136/bmjopen-2018021729).

Received 19 January 2018 Revised 22 March 2018 Accepted 27 April 2018

Check for updates

${ }^{1}$ Institute of Neuroscience and Physiology, Rehabilitation Medicine, Sahlgrenska Academy, University of Gothenburg, Gothenburg, Sweden

${ }^{2}$ Department of Public Health and Caring Sciences, Uppsala University, Uppsala, Sweden ${ }^{3}$ Institute of Internal Medicine, Department of Metabolism and Cardiovascular Research, Sahlgrenska University Hospital, Gothenburg, Sweden

${ }^{4}$ The Prehospital Research Center Western Sweden, Prehospen University College of Borås, Borås, Sweden

${ }^{5}$ Institute of Health and Care

Sciences, Sahlgrenska Academy, University of Gothenburg, Gothenburg, Sweden

Correspondence to

Adam Viktorisson;

adam.viktorisson@gu.se

\section{ABSTRACT}

Objectives The psychological outcome of out-of-hospital cardiac arrest (OHCA) has been studied more extensively in recent years. Still, not much is known about the wellbeing among OHCA survivors. In this retrospective crosssectional study, we aim to investigate post-OHCA wellbeing among patients with a good neurological outcome, 3 months after the cardiac event. To assess well-being, we analyse the frequency of anxiety, depression, posttraumatic stress disorder (PTSD) and health within this group. Further, we aim to evaluate the importance of five prognostic factors for post-OHCA well-being.

Methods Data collection took place between 2008 and 2012, and every OHCA survivor within one region of Sweden, with a cerebral performance category (CPC) score of $\leq 2$ at discharge, was asked to participate. Survivors were identified through the Swedish Cardiopulmonary Resuscitation Registry, and postal questionnaires were sent out 3 months after the OHCA. The survey included Hospital Anxiety and Depression scale (HADS), PTSD Checklist Civilian version (PCL-C) and European Quality of Life 5 Dimensions 3 level (EQ-5D-3L).

Results 0 298 survivors, 150 were eligible for this study and 94 responded. The mean time from OHCA to follow-up was 88 days. There was no significant difference between respondents and non-respondents in terms of sex, age, cardiac arrest circumstances or in-hospital interventions. 48 participants reported reduced well-being, and young age was the only factor significantly correlated to this outcome $(p=0.02)$. Women reported significantly higher scores in HADS $(p=0.001)$ and PCL-C $(p<0.001)$. Women also reported significantly lower $E Q-5 D$ index values $(p=0.002)$ and $E Q$-visual analogue scale scores $(p=0.002)$ compared with men.

Conclusion Reduced well-being is experienced by half of OHCA survivors with a CPC score $\leq 2$, and young age is negatively correlated to this outcome. The frequency of anxiety and PTSD is higher among women, who also report worse health.

\section{INTRODUCTION}

Out-of-hospital cardiac arrest (OHCA) is a severe condition, associated with an approximate survival rate of $10 \% .{ }^{1}$ However, the chance of survival has improved considerably over the last 20 years, implying that the group of OHCA survivors today is larger than ever. ${ }^{1}$ As the

\section{Strengths and limitations of this study}

- Data were collected from every out-of-hospital cardiac arrest (OHCA) survivor in a large region of Sweden and from a variety of hospitals, within the course of 4 years.

- A wide range of qualities related to emotional and physical well-being (anxiety, depression, post-traumatic stress disorder and health) were included in the survey.

- Only OHCA survivors with a good neurological outcome (ie, a cerebral performance category score of $\leq 2$ ) were included.

- The study population was drawn from one single region in Sweden.

- The European Quality of Life 5 Dimensions 3 level used in this study has recently been questioned concerning its ability to estimate health in OHCA populations.

brain is highly dependent on a constant blood flow, survivors may suffer cognitive impairment. ${ }^{2}$ Swedish studies have estimated that up to one-third of cardiac arrest (CA) survivors experience long-term neurological deficits. ${ }^{34}$ Problems with memory, attention and executive functioning are among the most common, although the frequency of neurological deficits varies greatly between different samples. ${ }^{5}$ A decrease in societal participation ${ }^{4}{ }^{6}$ and psychological sequelae like depression, anxiety and post-traumatic stress disorder (PTSD) has also been described following CA. ${ }^{7-10}$ Despite this, most studies investigating the outcome of CA report good health and high quality of life among survivors. ${ }^{11}$

Several prognostic factors have been shown to impact the neurological outcome after CA: gender, age, role of bystander, use of hypothermia and percutaneous coronary intervention (PCI) are among the most commonly reported. ${ }^{6} 12$ Less is known about prognostic factors regarding the psychological outcome, but receiving an implantable cardioverter defibrillator (ICD) and young age have been 
linked to psychological problems. ${ }^{813}$ However, the relation between the neurological and psychological problems following a CA is yet to be fully understood. ${ }^{14-16}$ In addition, it is likely that the ability to identify which patients may be at risk of emotional sequelae becomes even more important if the proportion of patients surviving OHCA continues to increase.

Even though psychological problems following CA have been increasingly highlighted in recent years, ${ }^{10} 14$ post-CA healthcare has been late to adapt. Scandinavian studies investigating CA follow-up show that the healthcare system often fails to provide cognitive, psychological and psychosocial support and rehabilitation. ${ }^{17} 18$ Our primary objective is to evaluate the frequency of wellbeing among OHCA survivors with a good neurological outcome 3 months after the cardiac event. In this study, well-being is defined as the absence of anxiety, depression and PTSD, as well as a health state in line with available data from a reference population in Sweden. Further, age, gender, time from CA to arrival of emergency medical service (EMS), treatment with hypothermia (at $33-34^{\circ} \mathrm{C}$ ) and ICD will be evaluated as prognostic factors for the prediction of post-OHCA well-being.

\section{METHODS}

\section{Study design and setting}

This was a retrospective cross-sectional study, executed with postal questionnaires sent to the included participants' homes 3 months after an OHCA. The setting was a region in Sweden with approximately 1.6 million inhabitants and 800 OHCAs annually; the 30-day survival rate of OHCA was about $10 \%$. Study participants were treated in nine different hospitals.

\section{Participants}

The study population comprised all OHCA patients who were alive 30 days after the event and who fulfilled the inclusion criteria of age $\geq 18$ years and a cerebral performance category (CPC) score $\leq 2$ at discharge from hospital, denoting good to moderate cerebral functioning. ${ }^{19}$ Exclusion criteria were severe medical complications and CA due to trauma, attempted suicide, drowning or intoxication. Patients not able to be identified within 3 months and patients not residing in the region were also excluded.

OHCA survivors were identified and enrolled from the national Swedish Cardiopulmonary Resuscitation Registry (SCRR), and every OHCA patient to whom CA treatment was initiated is included. To minimise missing entries, there was a strategically deployed nurse at each hospital, responsible for checking the entrances and recruiting patients not reported in the SCRR.

\section{Data collection and survey questionnaires}

A set of postal questionnaires, written in Swedish, were sent out to all included survivors during the enrolment period between 1 January 2008 and 31 December 2011.
If the patients did not wish to participate, they were encouraged to return the questionnaires and state why they declined participation. A reminder was sent if no response was received after 3 weeks. The postal survey included Hospital Anxiety and Depression scale (HADS), PTSD Checklist Civilian version (PCL-C) and European Quality of Life 5 Dimensions 3 level (EQ-5D-3L).

HADS is a self-administered screening instrument, divided into two subscales concerning depression and anxiety with a maximum score of 21 in each subscale. ${ }^{20}$ Scoring $>7$ is indicative of mild to moderate depression or anxiety; a score $>10$ indicates severe depression or anxiety. HADS has been shown to be a sensitive measurement for depression and anxiety, without somatic disorders as confounding factors. ${ }^{21}$

PCL-C is a self-administered screening instrument for PTSD regarding a predefined traumatic experience. The instrument comprises 17 items, in this study keyed to CA as the traumatic experience. Each item belongs to one of the following subscores: re-experience (1-5), avoidance (6-12), and hyperarousal (13-17). The respondent is asked to report on a scale from 1 ('not at all') to 5 ('extremely') how much he or she has been affected by each item during the last month. PCL-C is scored either as a cumulative score with a cut-off at 44 (range 17-85) or by evaluating the three separate subscores (symptom-criteria method). To meet PTSD criteria with the latter, at least one answer with a score $\geq 3$ for items $1-5$, three answers with a score $\geq 3$ for items $6-12$ and two answers with a score $\geq 3$ for items $13-17$ are required. PCL-C is validated and a reliable instrument for PTSD screening in different populations. ${ }^{22-24}$

EQ-5D-3L is a standardised survey designed to measure health. ${ }^{25}$ The instrument comprises five dimensions: mobility, self-care, usual activities, pain/discomfort and anxiety/depression. Each dimension has three levels: no problems, moderate problems and severe problems; the dimensions can be evaluated separately or combined as an index value. ${ }^{26}$ In this study, index values were calculated based on the UK EQ-5D index tariff. ${ }^{27}$ The survey also included a visual analogue scale (VAS)-scale where the patient assesses his or her self-perceived health on an analogue scale with endpoints labelled 'best imaginable health state' and 'worst imaginable health state'. EQ-5D-3L is commonly used as an outcome measure for cardiovascular disease, including CA. ${ }^{28}$

\section{Data analysis}

Statistical analyses were executed with IBM SPSS Statistics for Windows, V.24.0 (IBM, released 2016). All p values were two-tailed, and statistical tests were interpreted at a 0.05 significance level. Non-parametric tests were used for all group comparisons as all variables were non-normally distributed. The Mann-Whitney $\mathrm{U}$ test was applied for ordinal values and the $\chi^{2}$ test for nominal values. Spearman's rank correlation was used to explore correlations between background characteristics and the outcomes in terms of anxiety, depression, PTSD and health state. A 
binary logistic regression was performed to evaluate how age, time from CA to arrival of EMS, gender, treatment with hypothermia and ICD affected the outcome in terms of 'reduced well-being'. In these analyses, the outcome of well-being acquired an EQ-5D index value equal to or above the values from a Swedish reference population, adjusted to gender and age group, and scores non-indicative for anxiety ( $\leq 7$ in HADS anxiety scale), depression ( $\leq 7$ in HADS depression scale) and PTSD (by both cut-off and symptom-criteria method). The reference values regarding EQ-5D-3L were drawn from a random sample gathered 2001 in Stockholm County, Sweden $(n=3069){ }^{29}$

The participants were further divided into subgroups, with cut-offs based on the median age $(>64 / \leq 64$ years $)$ and the median time from CA to arrival of EMS $(>7 / \leq 7 \mathrm{~min})$, as well as groups divided by gender, treatment with hypothermia and ICD. Treatment with hypothermia was newly introduced during the recruitment period of this study, and there were no guidelines regarding who would receive treatment at the time. Consequently, there should be no predisposing difference between treated and untreated patients regarding hypothermia. The participants were also compared with reference populations regarding HADS and EQ-5D-3L. The mean HADS scores and percentage of participants scoring positive for anxiety and/or depression were compared with figures from a random sample gathered 1997 in Jämtland County, Sweden $(\mathrm{n}=624)^{30}$; the EQ-5D-3L dimensions were compared with the data from Stockholm County.

\section{Patient and public involvement}

There were no patients involved in the design or conduction of this study. The results will be reported to the Health \& Medical Care Committee of the Regional Executive Board, Region Västra Götaland, Sweden.

\section{RESULTS}

\section{Participants and group differences}

In all, 298 patients were alive at 30 days, 150 of whom were eligible for the study. In the group of excluded patients, 61 were either documented with an incorrect social security number, not able to be identified within 3 months or not residing in the region. The remaining 66 patients were considered too ill to participate or reported trauma, attempted suicide, drowning or intoxication as cause of the OHCA. Out of the 150 patients who received the questionnaire, 94 responded (63\% response rate) (figure 1). The mean time from OHCA to follow-up was 88 days $(\mathrm{SD} \pm 43)$. All the respondents (21\% women) answered the PCL-C and HADS questionnaire as well as the EQ-5D-3L dimensions; 86 of the respondents also evaluated their current health with the VAS (EQ-VAS).

There was no significant difference between men and women in the study population or between the respondents and non-respondents, regarding their background characteristics collected from the SCRR (table 1); neither was there any significant difference found regarding
CPC score between respondents and non-respondents. The study population's representativeness has also been investigated in a previous study, where no significant differences were found between the respondents and non-respondents in terms of age, CA circumstances and in-hospital interventions. ${ }^{31}$

\section{Well-being and prognostic factors}

Out of the 94 study participants, 46 reported well-being (49\%); among them, 5 were women (25\% of female participants) and 41 men (55.4\% of male participants), with a significant difference between the genders $(\mathrm{p}=0.02)$. A logistic regression analysis was performed for the prediction of a reduced sense of well-being 3 months after an OHCA (table 2). Women were found to have an OR of 3.19 compared with men; however, this was not significant. The only significant correlation found in the regression model was between age groups, where age correlated negatively to reduced well-being (table 2 ).

There was no significant difference in the subscores of HADS, PCL-C or EQ-5D-3L between subgroups divided by median age or median time from CA to arrival of EMS; neither was there any significant difference regarding treatment with hypothermia or ICD (table 3). However, younger age was slightly correlated to greater problems with PTSD $(\mathrm{r}=-0.3 ; \mathrm{p}=0.003)$ and anxiety $(\mathrm{r}=-0.24 ; \mathrm{p}=0.02)$.

\section{Anxiety and depression (HADS)}

Participants reported a mean score of $3.5(\mathrm{SD} \pm 3.5)$ in the depression subscale and $5.4(\mathrm{SD} \pm 4.6)$ in the anxiety subscale compared with corresponding figures of 3.98 $(\mathrm{SD} \pm 3.5)$ and $4.55(\mathrm{SD} \pm 3.7)$ in the reference population. Severe depression was less common among participants compared with the reference population; however, anxiety was more common, as seen in figure 2A. There was no significant difference between men and women in the depression scale of HADS; however, the mean anxiety score was significantly higher among women ( $\mathrm{p}=0.001)$, who also reported significantly more cases of anxiety $(\mathrm{p}=0.003)$ (table 4$)$.

\section{Post-traumatic stress disorder (PCL-C)}

Sixteen per cent of the participants scored positive for PTSD with the cut-off method and $21 \%$ when evaluated with the symptom-criteria method. The mean score was $31(\mathrm{SD} \pm 13)$ in the PCL-C questionnaire (table 4) and significantly higher among women $(\mathrm{p}<0.001)$. As seen in table 4 , there was also a significantly higher percentage of women scoring positive for PTSD when evaluated with both the cut-off method ( $\mathrm{p}=0.003)$ and the symptom-criteria method $(\mathrm{p}<0.001)$.

\section{Overall health (EQ-5D-3L)}

An EQ-5D index value indicative of full health ${ }^{32}$ was reported by 35 (37\%) of the participants compared with $45.6 \%$ in the reference population. Male participants represented $94 \%$ of those who reported full health. The mean EQ-5D index value was 0.79 ( $\mathrm{SD} \pm 0.24$; $\mathrm{SE}$ : 0.025$)$ in the study population compared with 0.84 (SE: 0.005) 


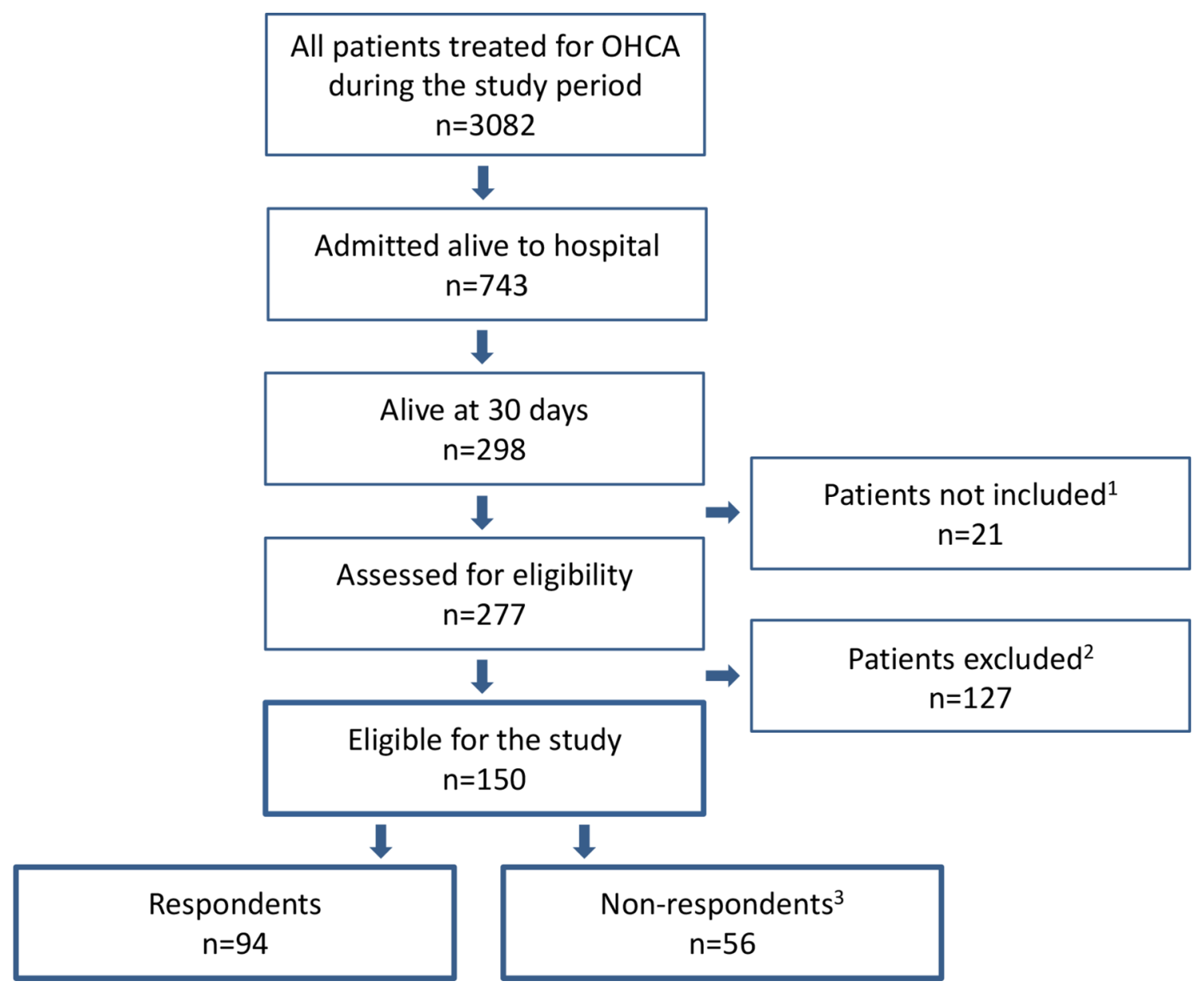

Figure 1 Flow chart describing the inclusion and exclusion process of the study population. OHCA, out-of-hospital cardiac arrest. 1 Of the not included patients 15 had a CPC-score > 2 and 6 died before follow-up. 2 Of the excluded patients, 61 were either documented with an incorrect social security number, not able to be identified within 3 months, or not residing in the region. The remaining 66 were considered too ill to participate or reported trauma, attempted suicide, drowning, or intoxication as cause of the OHCA. 3 Non-respondents included 29 patients lost to follow-up and 27 who declined participation; 13 patients declined without stating any reason, 5 due to poor health, and 9 due to "other reasons".

in the reference population. Participants reported more problems in every dimension except 'pain/discomfort' compared with the Swedish population-based data, as seen in figure 2B. Still, female participants reported more problems with pain and discomfort than the reference population. Female participants also reported significantly greater problems in every dimension except 'self-care', lower EQ-5D index values $(\mathrm{p}=0.002)$, as well as lower EQ-VAS scores compared with men $(\mathrm{p}=0.002)$ (table 4).

\section{DISCUSSION}

To evaluate the well-being among OHCA survivors with a good neurological outcome, we have analysed the frequency of anxiety, depression, PTSD and health state within this group, 3 months after the OHCA. We have also evaluated how age, gender, time from $\mathrm{CA}$ to arrival of EMS, treatment with hypothermia and ICD affect the outcome, in terms of well-being.

In this cross-sectional study, $49 \%$ of the OHCA survivors reported an EQ-5D index value in line with the reference population and no problems with anxiety, depression or PTSD, that is, were considered to experience well-being. To define health, WHO takes three forms of well-being into account: physical, mental and social. ${ }^{33}$ In order to approach the various forms of well-being described by WHO, we pooled three different questionnaires: HADS, PCL-C and EQ-5D-3L. In a recent article, researchers from the UK describe psychological well-being among CA survivors with the Depression Anxiety and Stress Scale $(\mathrm{DASS})^{14}$; the properties evaluated with the DASS are similar to those in HADS and PCL-C, which were used in this study. However, our definition should be considered more comprehensive since it also contains physical aspects of well-being in terms of mobility, experience of pain, hygiene and how well the patient can perform usual activities (eg, work, study, housework, family or leisure activities) found in the EQ-5D index value. Unlike HADS and PCL-C, the EQ-5D has no threshold value for the prediction of health; therefore, we used population averages, gathered from the general population in Stockholm County, as cut-off values for well-being regarding EQ-5D-3L. 
Table 1 Background characteristics of respondents, divided into men and women, and non-respondents in terms of age, cardiac arrest (CA) circumstances and in-hospital interventions

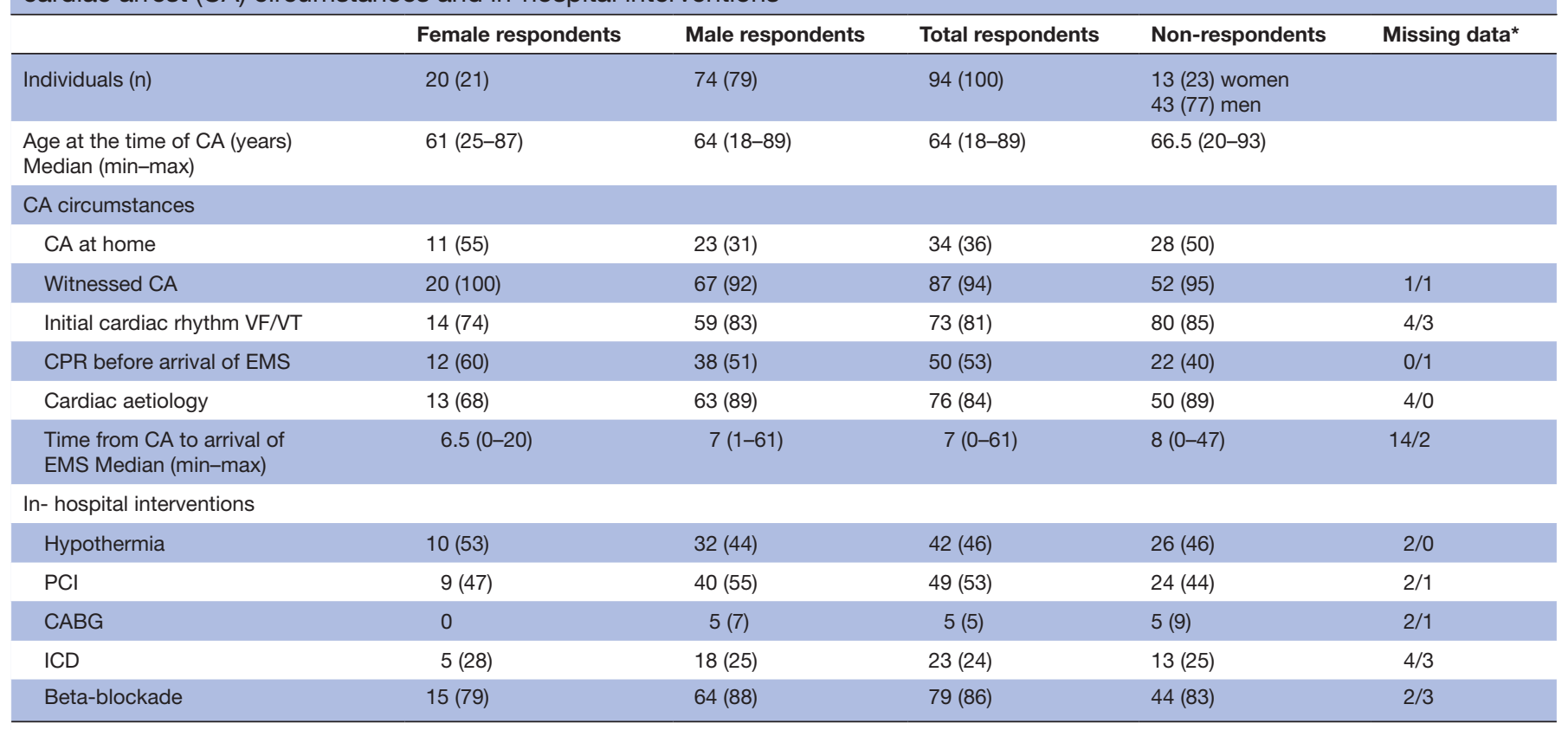

$\mathrm{n}(\%)$ unless otherwise stated.

*Number of patients where information is missing, among respondents/non-respondents.

CABG, coronary artery bypass grafting; CPR, cardiopulmonary resurrection; EMS, emergency medical service; ICD, implantable cardioverter defibrillator.; PCI,

percutaneous coronary intervention; VF, ventricular fibrillation; VT ventricular tachycardia.

In the logistic regression model, age was the only factor that correlated significantly with the outcome as a younger age was linked to a reduced sense of well-being. This result was expected since it has previously been reported that survivors of young age have greater problems with PTSD, anxiety and depression. ${ }^{813}$ Although studies investigating prognostic factors regarding conditions related to the psychological outcome of CA are scarce, receiving an ICD has previously been linked to problems with anxiety, depression and a poorer health state using HADS and EQ-5D. ${ }^{13}$ However, we were not able to find this correlation. Neither was there any significant difference between the sexes in our regression analysis, although women reported significantly more problems in the majority of instruments (HADS, PCL-C and EQ-5D-3L). It is possible to think that the outcome would have been different if the proportion of women had been larger; only 20 (21\%) out of the 94 participants were women.

We used previously published data, sampled from the general population in parts of Sweden, as references regarding HADS and EQ-5D-3L. There is no general reference data available regarding PCL-C as it requires that the user has been subjected to a traumatic experience, potentially yielding PTSD. However, the prevalence of PTSD in this study (16\% when evaluated with the cut-off method and $21 \%$ with the symptom-criteria method) is considerably lower compared with other studies investigating PTSD among patients surviving from acute life-threatening cardiovascular illness; for example, two studies from the UK in 2008 both found a prevalence of PTSD at $37 \% 3$ months after stroke. ${ }^{3435}$

Table 2 Logistic regression analysis for the prediction of reduced well-being $(n=77)$

\begin{tabular}{|c|c|c|c|c|c|c|}
\hline & $\beta$ & SE & Wald & df & P values & OR $(95 \% \mathrm{Cl})$ \\
\hline Female gender & 1.16 & 0.64 & 3.32 & 1 & 0.068 & 3.19 (0.92 to 11.1$)$ \\
\hline Age & -0.05 & 0.02 & 5.17 & 1 & 0.023 & 0.95 (0.91 to 0.99 ) \\
\hline $\begin{array}{l}\text { Time from CA to } \\
\text { arrival of EMS }\end{array}$ & 0.04 & 0.03 & 1.65 & 1 & 0.199 & 1.04 (0.98 to 1.1$)$ \\
\hline Use of ICD & 0.84 & 0.63 & 1.76 & 1 & 0.184 & 2.3 (0.67 to 8.04$)$ \\
\hline $\begin{array}{l}\text { Treatment with } \\
\text { hypothermia }\end{array}$ & 0.09 & 0.52 & 0.03 & 1 & 0.861 & 1.1 (0.39 to 3.06 ) \\
\hline Constant & 2.09 & 1.28 & 2.67 & 1 & 0.102 & - \\
\hline
\end{tabular}

$\mathrm{CA}$, cardiac arrest; df, degrees of freedom; EMS, emergency medical service; ICD, implantable cardioverter defibrillator. 


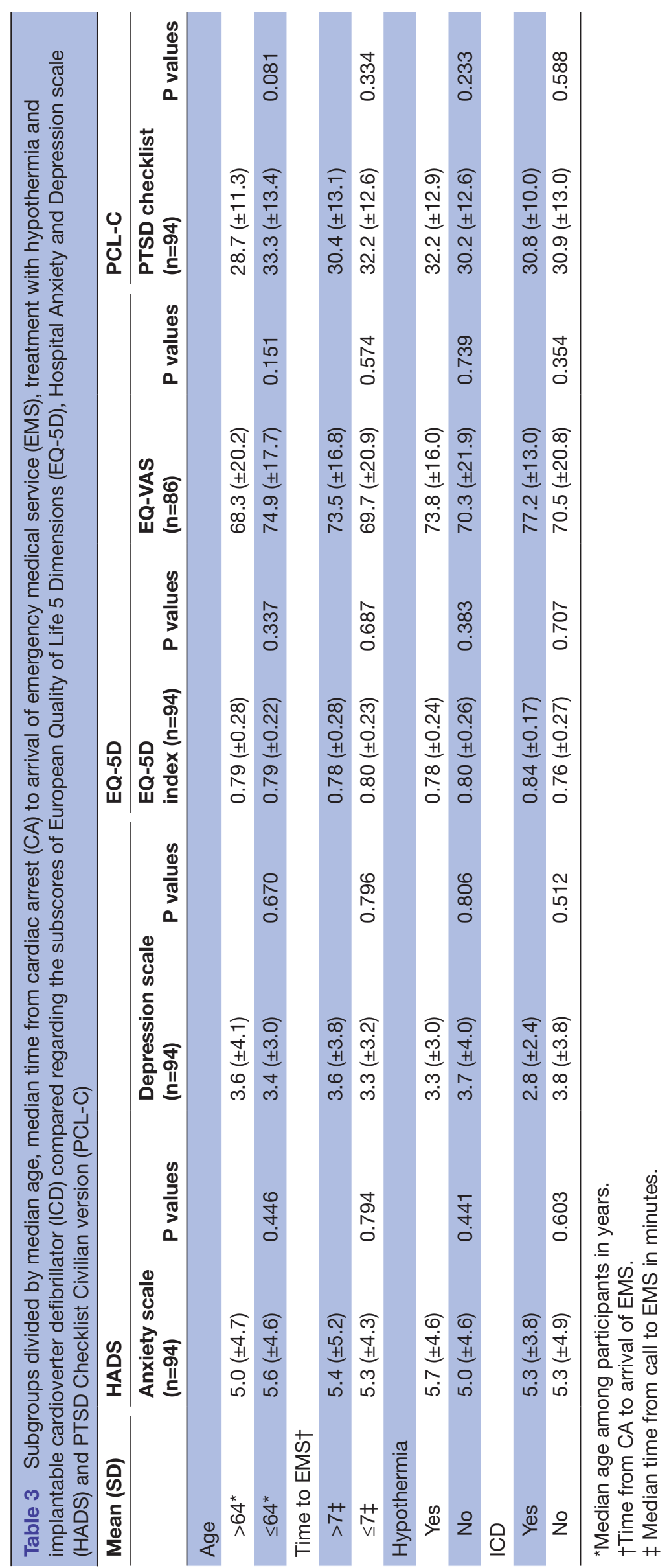

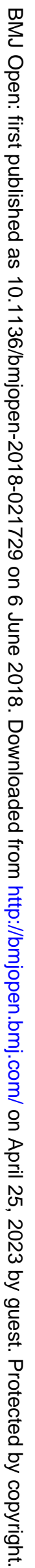


(A)

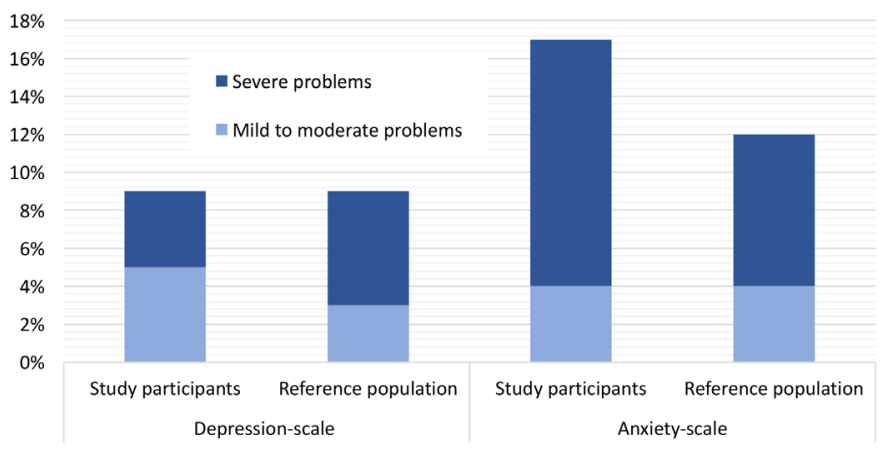

HADS - Study participants and reference population

(B)

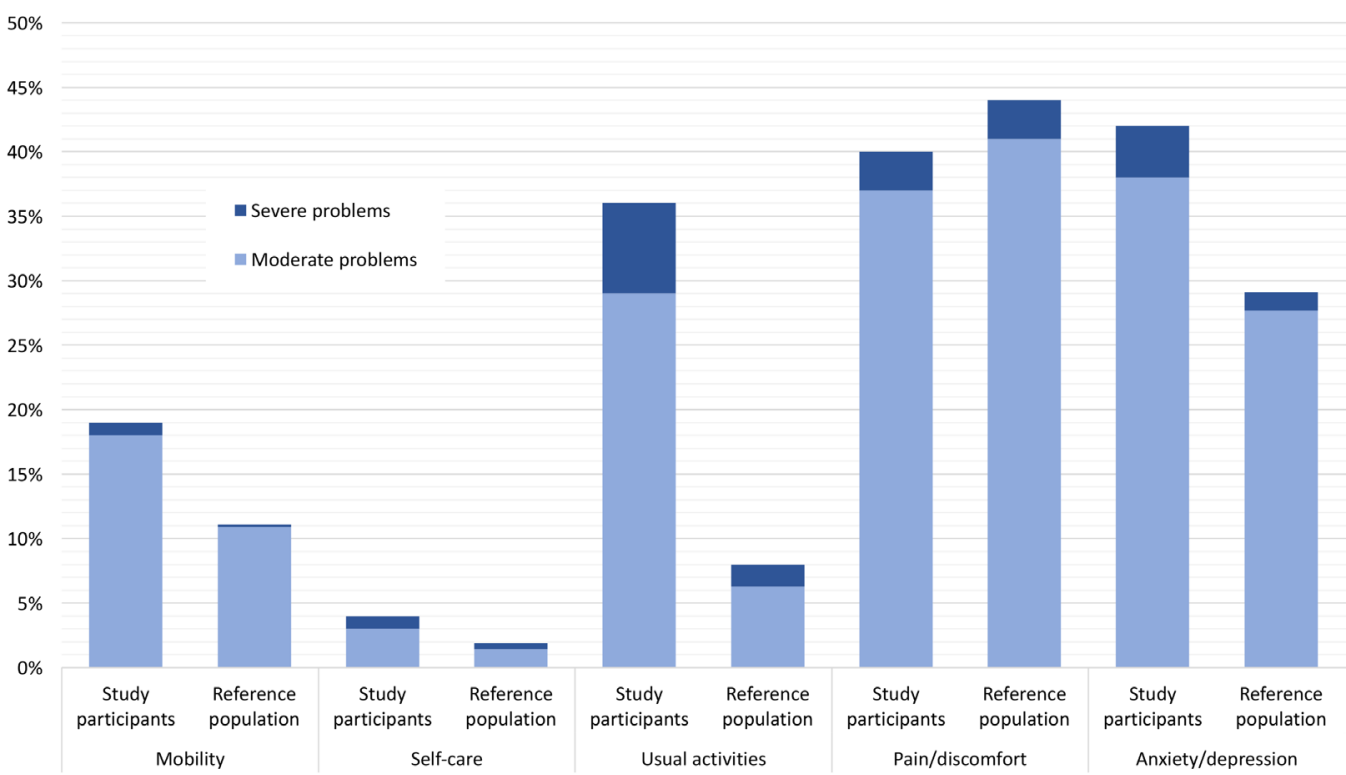

EQ-5D dimensions - Study participants and reference population

Figure 2 (A) Percentage of Hospital Anxiety and Depression scale (HADS) scores indicative for mild to moderate (score $>7$ ) or severe (score $>10$ ) problems with anxiety or depression reported by participants compared with the reference population in Jämtland County by Lisspers et al..$^{30}$ (B) Percentage of moderate and severe problems in the European Quality of Life 5 Dimensions (EQ-5D) reported by participants compared with the reference population in Stockholm County by Burström et al. ${ }^{29}$

One interesting finding in our study is that severe depression was less common among OHCA survivors compared with the reference population, although the frequency of overall depression was the same. This result was unexpected as many previous studies have reported much higher frequencies of depression during the first months post $\mathrm{CA}^{1636} \mathrm{~A}$ possible interpretation would be that the elation of having survived an OHCA could mask a depression, surveyed at 3 months after the cardiac event. Still, any interpretation of this finding is complicated by differences in sample recruitment as well as a relatively low response rate of $48 \%$ in the reference population. ${ }^{30}$ Further, studies investigating the long-term psychological outcome among CA survivors have found both higher and lower frequencies of depression compared with this study. ${ }^{6737}$

Among the predetermined subgroups, large differences were found between men and women; female participants reported significantly more cases of anxiety and PTSD as well as significantly higher scores in the anxiety scale of HADS and in PCL-C. Women were also found to have a significantly worse health state and self-assessed health (EQ-VAS). A few Swedish studies describing anxiety and health among CA survivors have previously reported similar results ${ }^{98}$; however, to our knowledge the significantly higher frequency of PTSD among female OHCA survivors found in this study has not been previously reported in any CA population. Women report more anxiety and poorer health compared with men in samples from the overall Swedish population as well ${ }^{29} 3940$; thus, it is possible that the differences regarding men and women found in this study are a manifestation of the already existing difference at population level. We found no significant difference regarding the sexes in the depression scale 
Table 4 Properties of Hospital Anxiety and Depression scale (HADS), PTSD Checklist Civilian version (PCL-C) and European Quality of Life 5 Dimensions 3 (EQ-5D) presented, divided and compared regarding gender

\begin{tabular}{|c|c|c|c|c|}
\hline Instruments & Total $(n=94)$ & Female $(n=20)$ & Male $(n=74)$ & P values (gender) \\
\hline \multicolumn{5}{|l|}{ HADS } \\
\hline \multicolumn{5}{|l|}{ Depression } \\
\hline Mean score (SD) & $3.5( \pm 3.5)$ & $4.8( \pm 3.9)$ & $3.2( \pm 3.4)$ & 0.074 \\
\hline$>7$ & $12(13)$ & $5(25)$ & $7(9)$ & 0.076 \\
\hline$>10$ & $4(4)$ & $2(10)$ & $2(3)$ & 0.198 \\
\hline \multicolumn{5}{|l|}{ Anxiety } \\
\hline Mean score (SD) & $5.4( \pm 4.6)$ & $8.6( \pm 4.7)$ & $4.5( \pm 4.2)$ & 0.001 \\
\hline$>7$ & $28(30)$ & $12(60)$ & $16(22)$ & 0.002 \\
\hline$>10$ & $12(13)$ & $7(35)$ & $5(7)$ & 0.003 \\
\hline \multicolumn{5}{|l|}{ PCL-C } \\
\hline \multicolumn{5}{|l|}{ Post-traumatic stress } \\
\hline Mean score (SD) & $31( \pm 13)$ & $42( \pm 14)$ & $28( \pm 10)$ & $<0.001$ \\
\hline PTSD by cut-off 44 & $15(16)$ & $8(40)$ & $7(9)$ & 0.003 \\
\hline PTSD by symptom criteria & $20(21)$ & $11(55)$ & $9(12)$ & $<0.001$ \\
\hline \multicolumn{5}{|l|}{ EQ-5D } \\
\hline \multicolumn{5}{|l|}{ Mobility } \\
\hline Moderate problems & $17(18)$ & $7(35)$ & $10(14)$ & \\
\hline Severe problems & $1(1)$ & $0(0)$ & $1(1)$ & $0.048^{*}$ \\
\hline \multicolumn{5}{|l|}{ Self-care } \\
\hline Moderate problems & $3(3)$ & $1(5)$ & $2(3)$ & \\
\hline Severe problems & $1(1)$ & $0(0)$ & $1(1)$ & $0.623^{*}$ \\
\hline \multicolumn{5}{|l|}{ Usual activities } \\
\hline Moderate problems & $27(29)$ & $10(50)$ & $17(23)$ & \\
\hline Severe problems & $7(7)$ & $2(10)$ & $5(7)$ & $0.014^{*}$ \\
\hline \multicolumn{5}{|l|}{ Pain/discomfort } \\
\hline Moderate problems & $35(37)$ & $11(55)$ & $24(32)$ & \\
\hline Severe problems & $3(3)$ & $1(5)$ & $2(3)$ & $0.041^{*}$ \\
\hline \multicolumn{5}{|l|}{ Anxiety/depression } \\
\hline Moderate problems & $36(38)$ & $14(70)$ & $22(30)$ & \\
\hline Severe problems & $4(4)$ & $2(10)$ & $2(3)$ & $<0.001^{*}$ \\
\hline \multicolumn{5}{|l|}{ EQ-5D index† } \\
\hline Mean score (SD) & $0.79( \pm 0.24)$ & $0.66( \pm 0.26)$ & $0.83( \pm 0.23)$ & 0.002 \\
\hline EQ-VAS & $n=86$ & $n=17$ & $n=69$ & \\
\hline Mean score (SD) & $71.9( \pm 19)$ & $61.2( \pm 15)$ & $74.6( \pm 19)$ & 0.002 \\
\hline
\end{tabular}

$\mathrm{n}(\%)$ unless otherwise stated.

${ }^{*}$ P-values for comparison between women with problems (moderate or severe) and men with problems (moderate or severe) in the EQ-5D dimensions.

†EQ-5D index values calculated with the UK EQ-5D index tariff.

PTSD , post-traumatic stress disorder; VAS, visual analogue scale.

of HADS, which confirms the results presented in a prior study. ${ }^{9}$ These findings stand in contrast to the overall population, where women are known to report more problems with depression. ${ }^{40}$

Before considering generalising our result to a broader OHCA population, it is important to remember that the sample in this study was drawn from one single region in Sweden and that only patients with a good neurological outcome (ie, a CPC score of $\leq 2$ ) were included. In addition, a large proportion of the excluded patients were so due to poor health; therefore, it is possible that our study population does not reflect the true majority of OHCA survivors. 


\section{Strengths and limitations}

One of the major strengths of this study is that we have been able to collect data from virtually every OHCA survivors in a large region, including a variety of hospitals, within the course of 4 years. This strength is mainly due to the composition of the research team, which included a nurse at each hospital, who could identify OHCA survivors and report missing patients in the SCRR. Further, the response rate reached a satisfying level of $63 \%$.

In addition to the retrospective nature of this study, the use of the SCRR should be considered a main methodical limitation as an investigative study has found the SCRR failing to report up to $25 \%$ of all OHCA cases. ${ }^{41}$ However, this can be considered partially compensated by the research team as efforts were made to find and contact non-included patients; a nurse was placed at each hospital in the region to identify every case of OHCA and report patients missing in the SCRR. The use of EQ-5D-3L may be considered another limitation. A recent study found EQ-5D lacking in the ability to differentiate between different degrees of good health due to high ceiling effects and thus underestimating the health of OHCA survivors ${ }^{42}$; this is important to remember when interpreting the results as our outcome in terms of well-being requires an EQ-5D-3L health status in line with a reference sample from the general population in Stockholm County. Further, only OHCA survivors with a good neurological outcome (ie, a CPC-score of $\leq 2$ ) were included in the study; this limits the possibility to apply the results on patients with poorer cognitive functioning and, therefore, the OHCA population at large.

Finally, the interpretation of well-being in this study is unique, although other research groups in the field have used similar definitions. ${ }^{143}$ Further, it is important to know that our results may not reflect every aspect of well-being. For example, some studies have reported a decrease in societal participation among OHCA survivors ${ }^{4}$; our outcome does not include social aspects of well-being, which should be considered a limitation when interpreting the results.

\section{CONCLUSIONS}

Half of the OHCA survivors with a good neurological outcome experience a reduced sense of well-being 3 months after the cardiac event. Among the potential prognostic factors evaluated, young age was the only one that correlated significantly to this outcome. The frequency of anxiety and PTSD is significantly elevated among female survivors, and women in this study also report worse overall health. These findings should be considered in the follow-up of OHCA patients. However, future studies are necessary to find further prognostic factors of importance for post-OHCA well-being and to highlight the various outcomes following an OHCA. Furthermore, clarity is needed as to whether the gender differences observed in this study are due to differences on a population level or generated by CA-related circumstances.
Acknowledgements The authors are grateful to all the nurses who contributed to the collection of data. They thank the personnel at Rehabilitation Medicine, Sahlgrenska Academy, for their kind help with statistical analysis and feedback. They also thank Dr Kate Bramley-Moore for language assistance.

Contributors All authors have made a substantial contribution to the conception and design of the study. KSS and ÅA initiated and planned the study, collected the data, assisted in the interpretation of the data, took part in drafting the manuscript and revised it for important intellectual content. UP and JH took part in the planning of the study, contributed with external expertise and have continuously revised the manuscript for important intellectual content. AV analysed the data and wrote the manuscript with input from all authors.

Funding The funding of this study was provided as a grant from the Health \& Medical Care Committee of the Regional Executive Board (VGFOUREG-78031), Region Västra Götaland, Sweden.

Competing interests None declared.

Patient consent Obtained.

Ethics approval Ethical permission was granted by the Regional Ethics Review board, Western Sweden (№. 465-07).

Provenance and peer review Not commissioned; externally peer reviewed.

Data sharing statement Due to ethical restrictions, data are available on request. Interested researchers may submit requests for data to the authors (ks. sunnerhagen@neuro.gu.se). According to Swedish regulation: http://www.epn.se/ en/start/regulations/, the permission to use data is only for what has been applied for and then approved by the Ethical board. To not follow the regulations is seen as scientific misconduct.

Open Access This is an Open Access article distributed in accordance with the Creative Commons Attribution Non Commercial (CC BY-NC 4.0) license, which permits others to distribute, remix, adapt, build upon this work non-commercially, and license their derivative works on different terms, provided the original work is properly cited and the use is non-commercial. See: http://creativecommons.org/ licenses/by-nc/4.0/

(c) Article author(s) (or their employer(s) unless otherwise stated in the text of the article) 2018. All rights reserved. No commercial use is permitted unless otherwise expressly granted.

\section{REFERENCES}

1. Strömsöe A, Svensson L, Axelsson ÅB, et al. Improved outcome in Sweden after out-of-hospital cardiac arrest and possible association with improvements in every link in the chain of survival. Eur Heart $J$ 2015;36:863-71.

2. Busl KM, Greer DM. Hypoxic-ischemic brain injury: pathophysiology, neuropathology and mechanisms. NeuroRehabilitation 2010;26:5-13.

3. Hofgren C, Lundgren-Nilsson A, Esbjörnsson E, et al. Two years after cardiac arrest; cognitive status, ADL function and living situation. Brain Inj 2008;22:972-8.

4. Lundgren-Nilsson $A$, Rosén $H$, Hofgren $C$, et al. The first year after successful cardiac resuscitation: function, activity, participation and quality of life. Resuscitation 2005;66:285-9.

5. Moulaert VR, Verbunt JA, van Heugten CM, et al. Cognitive impairments in survivors of out-of-hospital cardiac arrest: a systematic review. Resuscitation 2009;80:297-305.

6. Wachelder EM, Moulaert VR, van Heugten C, et al. Life after survival: long-term daily functioning and quality of life after an out-of-hospital cardiac arrest. Resuscitation 2009;80:517-22.

7. de Vos R, de Haes HC, Koster RW, et al. Quality of survival after cardiopulmonary resuscitation. Arch Intern Med 1999;159:249-54.

8. Gamper G, Willeit M, Sterz F, et al. Life after death: posttraumatic stress disorder in survivors of cardiac arrest-prevalence, associated factors, and the influence of sedation and analgesia. Crit Care Med 2004;32:378-83.

9. Lilja G, Nilsson G, Nielsen N, et al. Anxiety and depression among out-of-hospital cardiac arrest survivors. Resuscitation 2015;97:68-75.

10. Wilder Schaaf KP, Artman LK, Peberdy MA, et al. Anxiety, depression, and PTSD following cardiac arrest: a systematic review of the literature. Resuscitation 2013;84:873-7.

11. Elliott VJ, Rodgers DL, Brett SJ. Systematic review of quality of life and other patient-centred outcomes after cardiac arrest survival. Resuscitation 2011;82:247-56. 
12. Akahane M, Tanabe S, Koike S, et al. Elderly out-of-hospital cardiac arrest has worse outcomes with a family bystander than a non-family bystander. Int J Emerg Med 2012;5:41.

13. Thylén I, Dekker RL, Jaarsma T, et al. Characteristics associated with anxiety, depressive symptoms, and quality-of-life in a large cohort of implantable cardioverter defibrillator recipients. J Psychosom Res 2014;77:122-7.

14. Davies SE, Rhys M, Voss S, et al. Psychological wellbeing in survivors of cardiac arrest, and its relationship to neurocognitive function. Resuscitation 2017:111:22-5.

15. Polanowska KE, Sarzyńska-Długosz IM, Paprot AE, et al. Neuropsychological and neurological sequelae of out-of-hospital cardiac arrest and the estimated need for neurorehabilitation: a prospective pilot study. Kardiol Pol 2014;72:814-22.

16. Roine RO, Kajaste S, Kaste M. Neuropsychological sequelae of cardiac arrest. JAMA 1993;269:237-42.

17. Egerod I, Risom SS, Thomsen T, et al. ICU-recovery in Scandinavia: a comparative study of intensive care follow-up in Denmark, Norway and Sweden. Intensive Crit Care Nurs 2013;29:103-11.

18. Israelsson J, Lilja G, Bremer A, et al. Post cardiac arrest care and follow-up in Sweden - a national web-survey. BMC Nurs 2016;15:1.

19. Jennett $B$, Bond M. Assessment of outcome after severe brain damage. Lancet 1975;1:480-4.

20. Zigmond AS, Snaith RP. The hospital anxiety and depression scale. Acta Psychiatr Scand 1983;67:361-70.

21. Bjelland I, Dahl AA, Haug TT, et al. The validity of the hospital anxiety and depression scale. An updated literature review. J Psychosom Res 2002;52:69-77.

22. Blanchard EB, Jones-Alexander J, Buckley TC, et al. Psychometric properties of the PTSD checklist (PCL). Behav Res Ther 1996;34:669-73.

23. Ruggiero KJ, Del Ben K, Scotti JR, et al. Psychometric properties of the PTSD checklist-civilian Version. $J$ Trauma Stress 2003:16:495-502.

24. Weathers FW, Litz BT, Herman DS, et al, 1993. The PTSD Checklist (PCL): Reliability, validity, and diagnostic utility. Paper presented at the 9th Annual Conference of the ISTSS,San Antonio, TX

25. EuroQol Group. EuroQol-a new facility for the measurement of health-related quality of life. Health Policy 1990;16:199-208.

26. Burström K, Sun S, Gerdtham UG, et al. Swedish experience-based value sets for EQ-5D health states. Qual Life Res 2014;23:431-42.

27. Sullivan PW, Slejko JF, Sculpher MJ, et al. Catalogue of EQ-5D scores for the United Kingdom. Med Decis Making 2011;31:800-4.

28. Dyer MT, Goldsmith KA, Sharples LS, et al. A review of health utilities using the EQ-5D in studies of cardiovascular disease. Health Qual Life Outcomes 2010;8:13.
29. Burström K, Johannesson M, Diderichsen F. Swedish population health-related quality of life results using the EQ-5D. Qual Life Res 2001;10:621-35.

30. Lisspers J, Nygren A, Söderman E, et al. Hospital anxiety and depression scale (HAD): some psychometric data for a Swedish sample. Acta Psychiatr Scand 1997;96:281-6.

31. Axelsson ÅB, Sunnerhagen KS, Herlitz J. Representativity and comorbidity: Two factors of importance when reporting health status among survivors of cardiac arrest. Resuscitation 2016;101:44-9.

32. Szende A, Oppe M, Devlin NJ. EQ-5D value sets: inventory, comparative review and user guide: Springer, 2007.

33. World Health Organisation: WHO. Constitution of the world health organisation. Geneva: World Health Organisation, 1948.

34. Field EL, Norman P, Barton J. Cross-sectional and prospective associations between cognitive appraisals and posttraumatic stress disorder symptoms following stroke. Behav Res Ther 2008;46:62-70.

35. Noble AJ, Baisch S, Mendelow AD, et al. Posttraumatic stress disorder explains reduced quality of life in subarachnoid hemorrhage patients in both the short and long term. Neurosurgery 2008;63:1095-105

36. Kamphuis HC, De Leeuw JR, Derksen R, et al. A 12-month quality of life assessment of cardiac arrest survivors treated with or without an implantable cardioverter defibrillator. Europace 2002:4:417-25.

37. Ladwig KH, Schoefinius A, Dammann G, et al. Long-acting psychotraumatic properties of a cardiac arrest experience. Am J Psychiatry 1999;156:912-9.

38. Israelsson J, Bremer A, Herlitz J, et al. Health status and psychological distress among in-hospital cardiac arrest survivors in relation to gender. Resuscitation 2017;114:27-33.

39. Sun S, Irestig R, Burström B, et al. Health-related quality of life (EQ5D) among homeless persons compared to a general population sample in Stockholm County, 2006. Scand J Public Health 2012;40:115-25.

40. Johansson R, Carlbring P, Heedman $\AA$, et al. Depression, anxiety and their comorbidity in the Swedish general population: point prevalence and the effect on health-related quality of life. PeerJ 2013;1:e98.

41. Strömsöe A, Svensson L, Axelsson ÅB, et al. Validity of reported data in the Swedish cardiac arrest register in selected parts in Sweden. Resuscitation 2013;84:952-6.

42. Andrew E, Nehme Z, Bernard S, et al. Comparison of health-related quality of life and functional recovery measurement tools in out-ofhospital cardiac arrest survivors. Resuscitation 2016;107:57-64.

43. van Wijnen HG, Rasquin SM, van Heugten CM, et al. The impact of cardiac arrest on the long-term wellbeing and caregiver burden of family caregivers: a prospective cohort study. Clin Rehabil 2017;31:1267-75. 